\title{
Improving CAS Capabilities: New Rules for Computing Improper Integrals
}

\author{
José L. Galán-García, Gabriel Aguilera-Venegas, Pedro Rodríguez-Cielos, María Á. \\ Galán-García, Yolanda Padilla-Domínguez, Iván Atencia-Mc.Killop \\ University of Málaga \\ jlgalan@uma.es, gabri@ctima.uma.es, prodriguez@uma.es, magalan@ctima.uma.es, \\ ypadilla@ctima.uma.es, iatencia@ctima.uma.es
}

Ricardo Rodríguez

Technical University of Madrid

ricardo.rodriguez@upm.es

\begin{abstract}
There are different applications in Engineering that require to compute improper integrals of the first kind (integrals defined on an unbounded domain) such as: the work required to move an object from the surface of the earth to infinity (Kynetic Energy), the electric potential created by a charged sphere, the probability density function or the cumulative distribution function in Probability Theory, the values of the Gamma $(\Gamma)$ function (which is useful to compute the Beta $(\beta)$ function used to compute trigonometric integrals), Laplace and Fourier Transforms (very useful for example in Differential Equations), ...

Therefore, these applications need to deal with the following types of improper integrals:

$$
\int_{0}^{\infty} f(t) \mathrm{d} t \quad ; \quad \int_{-\infty}^{0} f(t) \mathrm{d} t \quad \text { and } \quad \int_{-\infty}^{\infty} f(t) \mathrm{d} t
$$

CAS (Computer Algebra Systems) use different rules for computing integrals. For example RUBI system, a rule-based integrator developed by Albert Rich [1], is a very powerful system for computing integrals using rules. But when computing improper integrals of the first kind, normally, the basic procedure is used (computing an antiderivative and some limits in infinite). But, what happens if an antiderivative or the limits do not exist? For example, for $\int_{0}^{\infty} \frac{\sin (a t)}{t} \mathrm{~d} t \quad ; \quad \int_{0}^{\infty} \frac{\cos (a t)-\cos (b t)}{t} \mathrm{~d} t \quad$ or $\quad \int_{-\infty}^{\infty} \frac{\cos (a t)}{t^{2}+1} \mathrm{~d} t \quad$ the antiderivatives can not be computed.

We will show some examples of improper integrals that CAS as Mathematica, Maple, Derive or Maxima can not compute. Using advance techniques as Laplace and Fourier transforms or Residue Theorem in Complex Analysis, we will be able to develop new rules schemes for these improper integrals. These new rules can extend the types of improper integrals that these CAS can compute.
\end{abstract}

\section{References}

1. A. RIch. Rule-based Mathematics. Symbolic Integration Rules. http://www. apmaths. uwo.ca/ arich/. 